\title{
EN TORNO A LA EMENDATIO DE CICERÓN, LUC. 26.85 EN LA EDICIÓN DE ANDREAS NAUGERIUS (VENECIA 1523) ${ }^{1}$
}

Antonio Moreno Hernández

UNED

anmoreno@flog.uned.es

\section{RESUMEN}

Análisis de la corrección a Luc. 26.85 que documenta la edición de la obra ciceroniana preparada por Andreas Naugerius (Venecia 1523).

Palabras Clave: Cicerón, conjetura, Andreas Naugerius.

\section{AbSTRACT}

Analysis of the correction to Luc. 26.85 that is preserved in the edition prepared by Andreas Naugerius (Venice 1523).

KeYwords: Cicero, Conjecture, Andreas Naugerius.

Cicerón, en el largo discurso de réplica a Lúculo en torno al debate sobre la fundamentación del conocimiento ( $L u c .64-147$ ), recoge un pasaje en 26.85 que la tradición incunable transmite así:

\footnotetext{
${ }^{1}$ Este artículo forma parte del Proyecto de Investigación FFI2011-23685, financiado por la Dirección General de Investigación, MINECO, dentro del Plan Nacional de Investigación.
} 
Dic mihi Lyssipus [Lisippus Venecia 1471, Reggio 1499] eodem aere, eadem temperatione, eodem caelo, aqua, ceteris omnibus, certum Alexandrum [Alexandriis Venecia 1471, Reggio 1499] eiusdem modi facere non posset. [posset? Venecia 1494, 1498] Qua igitur notione discerneres. [discerneres? Venecia 1494, 1498].

Todas las ediciones del cuatrocientos de la obra ciceroniana ${ }^{2}$ registran certum, siguiendo la tradición manuscrita mayoritaria ${ }^{3}$. La lectura se difundió en la primera edición del XVI, obra de Iodocus Badio Ascensio (Joost van Assche, 1462-1535), publicada en $1511(\text { Asc })^{4}$ :

\section{etiam fidifferat.Sed fi hominúfimilitudotanta effenon potett ne fignoriqudde. Dicmibl:

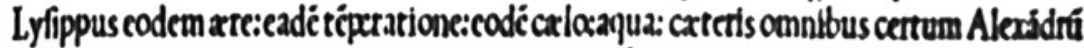

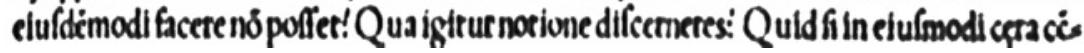

La misma lectura se reproduce en la siguiente de edición del mismo editor, en París $1521(\text { Asc. } 2)^{5}$ :

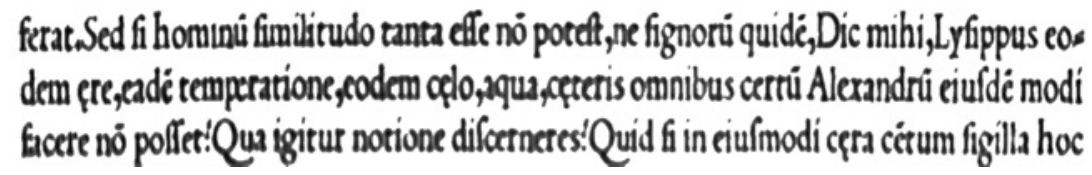

Sin embargo, en la edición que preparó Andreas Naugerius (Andrea Navagero, 1483-1529) para las prensas aldinas en 1523 (Ald. $)^{6}$, encontramos la corrección centum Alexandros:

${ }^{2}$ Roma 1471 (C. Sweynheim y A. Pannartz), Venecia 1494 (Christophorus de Pensis) y 1496 (Simon Bevilaqua), y Milán 1499 (Guillermus Le Signerre para Alexander Minutianus. En estas cuatro ediciones de Opera Philosophica ciceroniana se recogen los dos libros conservados de los Academica (Ac. I —Liber Primus de la segunda redacción, Academica posteriora_, y el Lucullus _ Liber secundus de la primera redacción, Academica priora-); el texto de Luc. se editó sin Ac. I en dos ocasiones: en Venecia 1471 (Vindelinus de Spira) y en Reggio d'Emilia 1499 (Bazalerius y Caligula de Bazaleriis).

3 Plasberg (1908, 1922), sobre la transmisión manuscrita del Luc., cf. Rouse 1981, 124-128, así como las recientes aportaciones de Malaspina 2011, 547-554; Malaspina, Borgna, Caso, Lucciano, Senore 2014, 589-620; Malaspina e.p.

${ }^{4}$ M. T. Ciceronis Opera Philosophica, quorum summam et seriem versa continebit pagina vaenudantur ab Iodoco Badio \& Ioanni Paruo, Lutetiae 1511. La edición ofrece en el Luc. muestra de una cierta actividad conjetural (cf. Hunt 1998, pp. 233-234); en N.D se aproxima a la edición de Minuatianus (Milan 1499), y en Fin. a la edición de Ulrich Zell (Colonia 1470). Sobre la labor de Ascensius como comentarista de textos clásicos, cf. White 2013. Actualmente las bibliotecas españolas albergan cuatro ejemplares de esta edición: (Madrid, Biblioteca Nacional, sign. 6/4733(1) y 2/6043(1); Madrid, Museo Arqueológico Nacional, sign. G'XVI-5-16; Cáceres. Biblioteca Pública del Estado «A. Rodríguez Moñino y María Brey», sign. 1/8999(1) (olim A/8999 - R. 6827). Las reproducciones de esta edición proceden del ejemplar conservado en Jena, Landesbibliothek, Bibliotheca Electoralis, Sign. 2 Phil.IX,7.

5 Opera Ciceronis Philosophica, apud Iodocum Badium Ascensium, Lutetiae 1521. Hays dos ejemplares conservados en España (CCPB000005274-4): Madrid, Biblioteca Nacional, sign. 2/14804(1), ejemplar que contiene los volúmenes de Opera Ciceronis philosophica y Opera epistolica; y Valladolid, Biblioteca Histórica de Santa Cruz (Universidad de Valladolid), sign., U/Bc BU 05615(1), encuadernados dos tomos en un solo volumen.

${ }^{6}$ M. T. Ciceronis De philosophia, Andrea Torresano de Asola in aedibuis Aldi, M. T. Ciceronis De philosophia I (mayo 1423): Ac. I, Luc., Fin., Tusc.. Cf. Renouard (I834, 97, 4-5); EDIT 16 (CNCE) 12220; USTC 822117; Adams 1967, C-1741. Se conserva dos ejemplares en los fondos españoles: Real Biblioteca 


\section{dic mabi, Lyfippus eodem are, cadem temperatione, codem celo, aqua, cateris omnibus, antum Alexan- dros ciufdem modi facere non poffit 'quaigzror no-}

La conjetura, que muestra la intensa actividad conjetural a la que somete Naugerius el texto ciceroniano ${ }^{7}$, se atribuye habitualmente por parte de la crítica a esta edición aldina, si viene no se puede descartar que proceda de la tradición manuscrita, toda vez que el impreso veneciano, que sigue fundamentalmente la edición de Ascensio de 1511, da muestras, para Ac. I, de haber incorporado, según Hunt (1998, pp. 234-235), variantes de uno o varios manuscritos del entorno de la llamada familia $\theta$, procedentes del norte de Italia, probablemente de Milán, en la primera mitad del s. XV, como el Dresd. 2 (Dresden, Sächsische Landesbibl. Dc 120) y sus descendientes, con los que Ald. comparte algunas variantes propias, si bien no revela una afinidad clara con ninguno de estos mss.

Es preciso sin embargo revisar los dos elementos de la conjetura atribuida a la edición aldina:

a) La corrección centum por certum - lectura que se documenta en la tradición mayoritaria a partir de los mss. más antiguos, (A V B), de ediciones incunables así como en las dos ediciones de Ascensio (Asc. y Asc. ${ }^{2}$ ) — responde a una confusión paleográfica habitual entre $n$ y $r$ que puede darse en diversas escrituras romanas, como la minúscula temprana (cf. Lindsay, 1896:87, «n, $\mathbf{r}$ in very early minuscule, e.g. Bachh. 793 terus for tenus; Curc. 26 sinit for sirit»), o la escritura insular (cf. Dumville 1995, 197-237), entre otras.

b) La corrección alexandros de Ald. en lugar de alexandrum (alexandrū $\mathbf{A}^{\mathbf{2}} \mathbf{B}$ edd. inc. Asc., Alexandriis en $\mathbf{V}^{2}$ ) fue asumida por editores como Davies (1725), Verburgius (1724), Olivetus (1740), Müller (1889) o Reid (1885), si bien resulta a nuestro juicio una intervención innecesaria, pues resulta más verosímil postular una mala interpretación de la abreviatura de alexandrū, que podría responder, en lugar al ac. sing., a una forma del ac. pl. en -us a la manera griega, como apunta Plasberg (1908 y 1922, cf. OLD s.v. Alexander).

La enmienda de certum en centum ofrece a nuestro juicio una solución muy plausible al pasaje, que se inscribe en el contexto de la argumentación escéptica sobre la imposibilidad de alcanzar una aprehensión fiable de objetos que se presentan como similares o idénticos, toda vez que resulta inviable la construcción de un conocimiento bien fundado cuando la percepción de los sentidos no se basa en una notio clara y distinta para cada objeto. Así ocurre en el caso de los gemelos Publio y Quinto Servilio Gémino,

del Monasterio de El Escorial, sign. 75-IV-10 — este ejemplar forma parte de los fondos que integraron la biblioteca del príncipe Felipe entre 1535 y 1559, germen de la biblioteca de El Escorial, junto con el segundo volumen de las Opera Philosophica editadas por la prensa aldina en 1523 (sign. 75-I-11) _; y Biblioteca de la Facultad de Teología de la Compañía de Jesús (Granada), sign. A-C43m. Edición digitalizada del ejemplar conservado en München, BSB, Res/A.lat.b 446-1.

7 Cf. Moreno 2014, 665-676, sobre la técnica conjetural de este humanista veneciano; cf. Luck 2002, 1-40, y $2005,155-224$. 
presentado en el parágrafo anterior (26.84), de manera que tras la similitud entre hombres, Cicerón aduce el parecido entre estatuas (sed si hominum similitudo tanta esse non potest, ne signorum), cuyas visiones (visa) no pueden distinguirse entre sí y por lo tanto no pueden dar lugar a percepciones ni juicios fiables. El ejemplo que aduce Cicerón evoca el privilegio, bien conocido en la época, que obtuvo Lisipo de Sición (segunda mitad del s, IV a.C.) para realizar estatuas de Alejandro, en virtud del decreto dictado por este estableciendo la prohibición de que nadie lo pintara salvo Apeles, ni nadie pudiera hacerle una escultura salvo Lisipo ${ }^{8}$.

La propuesta de Naugerius centum no fue considerada por algunos editores de las décadas siguientes, como Petrus Victorius, quien en su edición 1536 para las prensas venecianas de M. Antonio Giunta, consigna la lectio recepta de la tradición manuscrita e impresa previa, certum Alexandrum. Sin embargo centum ha sido asumida de forma generalizada por la crítica y los editores de la obra ciceroniana.

La aceptación de esta enmienda se advierte en la mayoría de las ediciones y en las traducciones, como ocurren con las del s. XIX de Nisard ${ }^{9}$, Charpentier ${ }^{10}$, Yonge ${ }^{11}$, y en las del s. XX, Millares Carlo ${ }^{12}$, Rackham $^{13}$, Schäublin ${ }^{14}$, y en las más recientes de Brittain $^{15}$ o Kany-Turpin ${ }^{16}$.

\section{REFERENCIAS BIBLIOGRÁFICAS}

Ediciones de Luc.

Ascensius, J. B. (1511). Opera Philosophica Ciceronis, Paris (= Asc.).

- (I 52 I ). Opera Philosophica Ciceronis, Paris $\left(=A s c .^{2}\right)$.

${ }^{8}$ Cf. Plutarco, Alex., 2.335 B-C; Cicerón, fam. 5.12,7; Horacio, Ep. 2.1,238; Valerio Máximo, 8.11; Apuleyo, Flor. 7.6 — donde se sustituye a Lisipo por Policleto, probablemente por error-; Plinio (Hist. Nat. 7.125) añade que sólo Pirgoteles estaba autorizado para grabar representaciones de Alejandro sobre piedras preciosas. Cf. F. de Olaguer-Feliú y Alonso $(2000,91)$.

9 Nisard (1864, 462): «Dites-moi si Lysippe avec le même métal, dans les mêmes proportions, avec le même air, la même qualité d'eau, toutes les autres conditions pareilles, ne pouvait pas faire cent Alexandres exactament semblables?» La versión de Acad. Traducción obra de M. A. Lorguet (Nisard 1864: 423-485).

${ }^{10}$ M. J. P. Charpentier (1872, 100): «Dites-moi: Lysippe ne pourrait-il pas, avec même bronze, même trempe, même air, même eau, et ainsi du reste, faire cent Alexandres semblables?»

${ }_{11}$ C. D. Yonge $(1891,61)$ : «Tell me, could not Lyssipus, using the same brass, the same composition of metals, the same atmosphere, water, and all other appliances, have made a hundred Alexanders exactly alike?»

12 Millares Carlo (1914: 96, y 1971: 98): «Dime, ¿no hubiera podido Sisipo (sic, corregido en "Lisipo” en la edición de México 1944: 143), con el mismo bronce, la misma aleación, el mismo aire, la misma agua y demás condiciones iguales, fabricar cien Alejandros idénticos?» (Millares sigue la edición de Müller 1908).

${ }_{13}$ Rackham (1933, p. 575: «Tell me, could not Lysippus, by means of the same bronze, the same blend of metals, the same graver and all the other requisites, make a hundred Alexanders of the same shape?»

${ }^{14}$ Schäublin (1995, 109-111): «Sage mir: hätte Lysipp mit derselben Bronze, mit derselben Mischung, mit demselben Stichel und Wasser und insgesamt unter denselben Bedingungen nicht hundert Statuen Alexanders von derselben Art herstellen Können?»

${ }^{15}$ Brittain (2006, p. 49): «Are you saying that Lysippus couldn't have made a hundred Alexanders just like one another, if he used the same bronze, the same process, the same tool, etc.? (sigue fundamentalmente el texto de Plasberg (Brittain 2006, XLIII).

${ }^{16}$ Kany-Turpin (2010: 213): «Dis-moi: Lysippe, avec le même bronze, le même mélange, le même ciseau et tout le necessaire n'aurait-il pu sculpter cent Alexandres de même forme?». Sigue a Plasberg (Kany-Turpin 2010: 67). 
BAiter, J. G. (1863). Cicero, M. T.: Academicorum quae supersunt, Leipzig, Teubner.

Brittain, CH. (2006). On Academic Scepticism, Indianapolis Cambridge.

Charpentier, M. J. P. (1872). Ouvres Complètes de Cicéron: Les Académiques, Des Vrais et des vrais maux, Les Paradoxes, trad. (Ac. ) M. Delcasso, J. P. Charpentier, Paris.

Colineus, S. (1535). M. Tullii Ciceronis, Academicarum quaestionum libri, primus et quartus, Paris.

Curio, V. (1525). Academicarum Quaestionum liber quartus et fragmentum primi, Basel.

Davies, J. (1725-1736). M.T. Ciceronis Academica, Cambridge.

DuRAND, D. (1740). M.T. Ciceronis opera, London.

ERnesti, J. A. (1830). M.T. Ciceronis Opera Philosophica, London.

- (I83I). Clavis Ciceroniana, Halle.

Görenz, I. A. (1810). M.T. Ciceronis Philosophica Omnia (vol. II), Leipzig (Whitefish, Mont., 2009).

Gruter, J. (1618). M.T. Ciceronis opera, Hamburg.

HaLm, K. (1861). M.T. Ciceronis Opera quae supersunt omnia (ex recensione J. C. Orellii, J. G. Baiter, K. Halm), vol. IV, Zurich.

Hülsemann, Fr. (1806). M.T Ciceronis Academica, Magdeburg.

Kany-Turpin, J. (2010), Cicéron, Les Académiques, Paris.

KLotz, R. (1854). Cicero, Academicorum ad M. Varronem libri duo, Leipzig.

Lambinus, D. (1565). M.T. Ciceronis Opera omnia, Paris.

LeClerC, J. V., Bouillet, M.N. (1828). M.T.C. Opera philosophica, vol. I, Paris.

Honoré, F. L' (1689). Opera Philosophica, Paris.

Manutius, A. (1523). M.T. Ciceronis opera philosophica, vol. I, Venecia (= Ald.).

Manutius, P. (1541). M.T. Ciceronis opera philosophica, Venecia.

Millares Carlo, A. (1914). Cuestiones Académicas, Barcelona (México 1944; Madrid 1971).

MülleR, C. F. W. (1889). M.T. Ciceronis Scripta quae manserunt omnia, vol. IV.1, Leipzig.

NisARd, M. Oeuvres complètes de Ciceron (avec la traduction en français), III, Paris.

Olivetus, J. (1740). Tullii Ciceronis opera, cum delectu commentariorum, vol. II, Paris.

Orelli, J. C. (1827-1828), M.T. Ciceronis opera, Zürich.

Plasberg, O. (1908). M.T. Ciceronis Paradoxa Stoicorum, Academicorum reliquiae cum Lucullo, vol. I., Leipzig.

Plasberg, O. (1922). M.T. Ciceronis Academicorum reliquiae cum Lucullo, Leipzig (reimpr. Stuttgart 1980, 1996).

Rackham, H. (1933). Cicero, De natura deorum, Academica, Cambridge Mass.- London (reimpr. 1967)

Ranitz, A. C. (1809). De libris Ciceronis Academicis comentatio, Leipzig.

ReID, J. S. (1874). M.T. Ciceronis Academica (The Text Revised and Explained), London.

ReID, J. S. (1885). M.T. Ciceronis Academica (The Text Revised and Explained), London (reimpr. Hildesheim 1966).

RiHEL, W. (1549). M.T. Ciceronis librorum philosophicorum volumen primum, Strasbourg.

SchäUblin, Ch. (1995). M.T. Cicero, Akademische Abhandlungen. Lucullus, Hamburg.

Stephanus, R. (1536). MT. Ciceronis Opera, Paris.

Talaeus, A. (1550). Ciceronis Academica, Paris.

Verburgius, I. (1724). MT. Ciceronis Opera, vol. VIII, Amsterdam

VICTORIUs, P. (1536). M.T. Ciceronis opera philosophica, Venecia.

Victorius, P. (1538). Explicationes suarum in Ciceronem castigationum, Paris.

Yonge, C. D. (1891). The Academic Questions, Treatise de Finibus, and Tusculan Disputations of M.T. Cicero, London. 


\section{BIBLIOGRAFÍA}

Adams, H. M. (1967). Catalogue of Books Printed on the Continent of Europe, 1501-1600, Cambridge.

Dumville, D. N. (1995). «The early medieval Insular churches and the preservation of Roman literature: towards a historical and palaeographical reevaluation», O. Pecere, M.D. Reeve (eds.), Formative stages of classical traditions: Latin texts from Antiquity to the Renaissance, Spoleto, 197-237

Hunt, T. J. (1998). A textual History of Cicero's Academici Libri, Leiden-Boston-Köln.

Lindsay, W. M. (1896). An Introduction to Latin Textual Emendation based on the text of Plautus, London.

LucK, G. (2002). «Ovid, Naugerius and we, or: how to create a text», ExClass 6, 2002, 1-40.

- (2005). «Naugerius's Notes on Ovid's Metamorphoses», ExClass 9, 2005, 155-224.

Madvig, J. N. (1826). Emendationes in Ciceronis libris De legibus et Academica, Kopenhagen.

- (I 839). M.T. Ciceronis de finibus bonorum et malorum, Kopenhagen.

Malaspina, E. (2011). «Primae notulae ad Luculli Ciceroniani recentiores recensendos», en A. Balbo, F. Bessone, E. Malaspina (a cura di), «Tanti affetti in tal momento». Studi in onore di G. Garbarino, Alessandria, 547-554.

Malaspina E., Borgna, A., Caso, D., Lucciano, M., Senore, C. (2014). «I manoscritti del Lucullus di Cicerone in Vaticana: valore filologico e collocazione Stemmatica», Miscellanea Bibliothecae Apostolicae Vaticanae, 20, 589-620.

Malaspina, E. (e.p.), «La tradizione manoscritta del Lucullus di Cicerone: dal Corpus Leidense a William of Malmesbury e alla fortuna in età umanistica», en M. MARTINHO, I. TARDIN CARDoso (eds.), Ciceronianissimos II, São Paulo, e.p.

Moreno Hernández, A. (2014). «A propósito de Cicerón, Luc. 11.34; 23.72 y 25.81, y la técnica conjetural de Andreas Naugerius (Venecia 1523)», J. M. Baños Baños, Mª F. del Barrio Vega, $\mathrm{M}^{\mathrm{a}}$ Teresa Callejas Berdonés, A. López Fonseca (eds.), Philologia, Universitas, Vita. Trabajos en honor de Tomás González Rolán, Escolar y Mayo Editores, Madrid, 2014, 665-676.

Olaguer-Feliú y Alonso, F. de (2000). Aproximación a la personalidad de Alejandro Magno y a su influencia en el Arte, Madrid.

Renouard, A.-A. (1834). Des annales de l'imprimerie des Aldes, Paris (New Castle 1991).

Rouse, R. H. (1981). «Cicero, Academica Priora», Reynolds, L. D. (ed.), Texts and transmission, Oxford, 124-128.

Vecce, C. (1996). «Bembo e Cicerone», Ciceroniana, Atti del IX Colloquium Tullianum, Roma, 147-159.

White, P. (2013). Jodocus Badius Ascensius. Commentary, Commerce and Print in the Renaissance, Oxford. 